\title{
Addiction Research
}

\section{Moral Resistance in Treatment of Post-Traumatic Stress Disorder}

\author{
Mahmud Said Ph.D*
}

Specialist in Educational and Clinical Psychology, Supervisor in Educational Psychology, Therapist and trainer in Psychological Trauma in Traumatic Incident Reduction - TIR.

\section{${ }^{*}$ Correspondence:}

Mahmud Said, Specialist in Educational and Clinical Psychology, Supervisor in Educational Psychology, Therapist and trainer in Psychological Trauma in Traumatic Incident Reduction - TIR.

Received: 24 August 2021; Accepted: 20 September 2021

Citation: Said M. Moral resistance in treatment of Post-Traumatic Stress Disorder. Addict Res. 2021; 5(1): 1-6.

\section{ABSTRACT}

This case study illustrates with a detailed report the treatment of a person with post-traumatic stress disorder (PTSD). The therapist noted that the patient did not improve despite all the necessary psychological interventions taken, the patient's request for treatment, and his commitment to the treatment. Enquiry revealed that the patient unconsciously preferred to continue suffering and feeling guilty towards his deceased so. The patient was suffering in order to preserve his loyalty to his deceased son, a phenomenon which we name moral resistance. PTSD Symptom Scale Interview (PSSI) was applied pre and post treatment and in follow-up. The Traumatic Incident Reduction (TIR) was the main therapeutic strategy, as well as Rational Emotive Behavior Therapy (REBT). The study found significant effectiveness of TIR and REBT in treatment of moral resistance aaccording to the PSSI scale. The total score decreased from 43, which indicated a very severe level of the disorder, to 10 a week after commencement of the treatment, and to 7 after a month. Scores of 4 was kept constant in 3- and 6-months follow-up.

\section{Keywords}

PTSD, TIR, REBT, Moral Resistant.

\section{Posttraumatic stress disorder (PTSD)}

Post-traumatic stress disorder (PTSD) is defined as "a psychiatric disorder that can develop following the direct, personal experiences or witnessing of a traumatic event, often life-threatening and characterized by re-experiencing, avoidance or numbing and hyperarousal" [1]. PTSD develops through the subject's exposure to an unexpected traumatic event such as wars or unexpected loss of a loved one in a traumatic event [2]. Due to the Diagnostic and Statistical Manual of Mental Disorders (DSM-5) causes to PTSD include exposure to a traumatic event which occurred to a close family member or a close friend, and the event must have been accidental [3]. The estimated lifetime prevalence of PTSD in community samples ranges between five to ten percent [4].

Signs and symptoms of clinically developing significant psychological distress include avoidance, hyperarousal, and reexperiencing related to the event [5]. Not all people who have been traumatized need medical or psychological treatment. Necessity of treatment depends on the severity, duration and continuity of reexperiencing, avoidance and arousal symptoms, and impairment in functioning and as well as social involvement, all of which should be reported for at least six months after the traumatic event. Early treatment is recommended as soon as signs and symptoms of PTSD are reported [6]. Additional other signs and symptoms occur include nightmares, flashbacks, intrusive thoughts, guilt feeling, inappropriate levels of fear and powerlessness joined by functional impairment [7]. Another complication of losing family member may be grief development if not treated [8].

Mental health is affected by stigma and discrimination for long time. This problem induces suffering and burden to clients and their families and leads to delayed treatment [9]. Victim's knowledge and perception of the traumatic event and the consecutive symptoms affect the way they deal with, including applying to treatment. Cognitive perception has a high effectiveness in adopting the appropriate behavior and positive thinking regarding traumatic event [10]. The clinician should help the patient to be aware of the emotions associated with the event, whether it is fear, anxiety, shame, guilt, anger, or sadness [11]. Patient's acceptance of these emotions plays the main role in avoiding negative internal experiences such as thoughts, emotions, memories, and sensations [12]. 
The recovery from trauma depends on the nature of the traumatic event, social support and on patient's personality [13]. People characterized with positive attitudes are better in eliminating the effects of stress, and have better ability to control their thoughts and behavior, leading to relief from the trauma effects [14]. Cognitive-behavioral therapy and exposure therapy are effective in treating trauma, exposure therapy is regarded being the first line in the treatment of trauma [15].

\section{Traumatic incident reduction (TIR)}

Traumatic Incident Reduction (TIR) was introduced by Gerbode (1988). This method is used in the treatment of PTSD by recalling the important information about the nature and consequences of the traumatic events [16]. TIR is a brief, clear, memory-based, most similar to imaginal flooding interventions, and focusing on memory rather than on symptom management [17]. The goals of TIR as was conducted by Gerbode (1988), were increasing re-experiencing of negative emotions related to the trauma, increasing deliberate cognitive processing of the trauma, and creating narrative memory of the trauma [18].

When conducting TIR the therapist should follow the following outline: (a) give informed consent, (b) complete pretest measures, (c) have a one-on-one orientation interview to learn the nature of the trauma and the roles that they and the mental health practitioner would play, (d) receive a session of TIR, (e) complete a debriefing session, and (f) complete posttest and follow-up measures [19]. The client is directed to imagine the traumatic event without the use of verbal expression, followed by repeating telling the event. The effectiveness of using this method depends on the client's ability to imagine [20]. In the sessions the client describes a particular negative feeling, attitude, or thinking, which serve to identify specific incidents for resolution. The therapist reviews the client's feelings. Then the client is able to talk calmly about the traumatic event without the appearance of the symptoms [21].

\section{Rational emotive behavior therapy (REBT)}

The cognitive interpretation of a traumatic experience depends on the sequence of events. A negative interpretation of the traumatic event is followed by irrational behavior. Irrational beliefs and behaviors are expressed in many forms such as a sense of unreality about the traumatic event negative perception of self, life and future, catastrophic misinterpretation of grief reactions, and avoidance behavior accompanied by anxiety [22]. REBT originated from rational therapy which developed to RationalEmotive Therapy and in 1990 became Rational Emotive Behavior Therapy. There are many similarities between cognitive-behavioral therapy and REBT but recently the therapy moved away from its cognitive roots [23]. REBT deals with irrational beliefs in a broader sense than cognitive behavior therapy in the treatment of posttraumatic stress responses [24]. Behavioral, cognitive and emotive techniques involved in the practice of this therapy in order to promote change of negative thoughts, emotions, and behaviors in a positive manner [25].

ABC model shows the emotional interaction in which " $A$ " refers to activating the event, "B" refers to the belief or interpretation of the activated event, and " $\mathrm{C}$ " refers to the consequent emotional, physiological, and/or behavioral responses [26]. The A-B-C model is considered a cornerstone in the theory and practice of REBT, the consequences $(\mathrm{C})$ of disturbances in thoughts and emotions are related to people's own irrational beliefs (B) and not by the activating event (A) [27].

REBT techniques are useful for therapists working with parents and families by focusing on their negative thoughts and trying to change them, enhancing emotional performance, and increasing their ability to make effective behavior management decisions [28].

In the grief processes, according to REBT interpretation, the maladaptation occurs when traumatic death causes negative thoughts which are called irrational beliefs. There is a disparity with a person who is traumatized between adaptive rational belief and maladaptive irrational belief, that depends on thoughts and emotions [29]. Psychologists focus on three levels of client response during sessions: behaviors (i.e., observable and measurable client reactions), cognitions (.i.e., information processing) and subjective experience (i.e., feelings and emotions) [30]. Recently a number of evocative-emotive techniques used in REBT therapy such as rational emotive imagery, shame attacking exercises, role-playing, reverse role playing, forceful coping statements, unconditional acceptance of clients were added [31]. The therapist should use open-ended questions in sessions to assess thoughts and emotions, intervention process, and future performance [32].

\section{Moral Resistance}

We did not find previous studies or references on moral resistance, which motivated us to write this paper in order to highlight this important problem and provide solutions and recommendations.

It is important to make a distinction between moral resistance and Client engagement. These concepts could be difficult to be distinguished on the basis of clinical observation without long-term systematic evaluation, especially in the case of our patient who looked for treatment and was committed to, but unconsciously did not follow the instructions. It was eventually found that he believed that relieving his distress would mean him being unfaithful to his late son, thus he feared feeling guilty if his suffering ends.

Client engagement in treatment is one of the most significant aspects of the therapeutic process. It reflects the level of healthy attachment a client creates with the therapist and directly impacts the prognosis. In this article, we will take an in-depth look into the core aspects of moral resistance and discuss ways to solve it. We have noticed while working as a trauma therapist, that while the client came willingly to seek treatment for his traumatic and severe experience, and the therapist did his best to provide all necessary interventions, however the treatment did not reach its goals satisfactorily.

It seems as if the client is unconsciously not willing to cooperate with the therapist. On the one hand, he asks for help to alleviate his pain and suffering from trauma and loss and desires bringing about the desired change and is committed to attending the sessions. On 
the other hand, he unconsciously refuses to follow the instructions. While he consciously declared his wish to feel better and readjust to life, he unconsciously refrained from ending his suffering, because of his devotion to his lost son.

In medicine, the term 'resistance to treatment' usually serves to define patients failing to respond to a standard form of treatment. Similarly, treatment-resistant PTSD applies to those patients who do not resolve symptoms although they have been treated adequately with pharmacological, non-pharmacological, or combined treatment approaches. The definition may vary widely and there is no universally accepted definition for how long a patient should be treated with no progress that would be accepted as treatment-resistant. However, considering the onset of PTSD and symptoms, it might be accepted that twelve months of treatment failure might be an acceptable definition. This, of course, depends on adequate treatment trial and the specific expectations for treatment outcomes. In theory, treatment-resistant PTSD may apply to describe patients with an initial response to the therapeutic approach but with no significant improvement achieved, with respect to resolving symptoms, improving sense of self-esteem, and functioning [33].

\section{The treatment}

The subject of this report is a 56 year old man. In the initial interview we found him to meet the DSM-5 [3] criteria for active PTSD while his main complains were insomnia and sever psychological distress lasting for more than one year. He reported that he started experiencing these symptoms since he lost his elder son. The family was Israeli Palestinians. This population is not obliged to serve in the armed forces, according to the Israeli law, but his son insisted to recruit, against his father will. The son was killed while serving in the occupied territories in the area of the Gaza Strip, shot by a Palestinian sniper. The client was very traumatized when he heard his son was killed.

A diagnosis of PTSD was determined by means of psychiatric interview. The client signed a consent form for participating in psychotherapy. The therapist explained the procedures of the sessions in detail and the initial treatment session was scheduled.

After providing all the necessary psychological interventions, the therapist noticed that the patient did not improve despite his commitment to the sessions and his request for treatment. Through psychiatric assessment, the therapist discovered that the patient was not cooperative and believed that if he gets rid of his suffering, he will be unfaithful to his deceased son.

In this study, we used two approaches for treatment of the PTSD: Traumatic incident reduction (TIR), and Rational emotive behavior therapy (REBT).

The PTSD Symptom Scale Interview (PSSI) [34] had been used to assess symptoms related to the identifiable traumatic event and current symptoms of PTSD for goals of diagnosis as well as following the progression of the treatment. PSSI scale consists of
17 items representing three domains: Re-experiencing, Avoidance, and Increased arousal [35]. The scale was translated to Arabic Language by a linguist and then sent to three Arab native speakers with high level of knowledge of English. The PSSI scale was applied pre-treatment and post-intervention at different intervals: after one week, one month, three months, and six months. When the therapist got the impression of lack of progression in the treatment, he added an open, unstructured conversation in order to detect potential causes.

As the client was diagnosed with PTSD, he received information regarding the PTSD syndrome and the therapeutic procedures that are to be taken. In the beginning of the treatment, we invested in creating therapeutic bond and establishing trust. The client was asked to select one traumatic event, and he chose the situation of him being informed about the death of his son. The therapist asked the client to imagine the event and what happened without talking and then to recount what happened aloud, and re-experiencing the event again and again. At that time of commencement of the treatment the therapist got the impression that the client was cooperating and the therapeutic plan was going in the required direction.

In the following sessions, the client repeated the event several times. He began with brief words and emotions, gradually talking more and showing more emotions, and reviving the event. However, a significant improvement in his condition was not observed, in contrast to what was expected when compared to what had been seen with former clients as well as what is expected from reports in literature [36].

The therapist asked the client whether he felt his emotions to improve or worsen and the client replied that it felt better, however this positive response did not match the therapist's impression of the client behaviour and gestures. The session went on with the procedure described above, the therapist taking serious efforts to follow faithfully the TIR protocol, with no apparent difference. The therapist asked again whether he felt better or worse, and the client said he felt better. Noticing the discrepancy between the observed and the reported outcome, the therapist became convinced that the therapeutic process did not proceed correctly. The therapist shared his impression with the client, who responded by saying that he was completely cooperative with what he was asked to do.

At that stage the therapist took a decision to intermit the continuation of the TIR protocol. He asked the client if he felt that he was genuinely willing the treatment? which the patient nodded with a decisive "Yes". The therapist went on asking whether the client was fully honest concerning his inner feelings. Again, the client answered "yes", but at that time there was some hesitant tone in his voice. The therapist insisted again whether the client was definite about it. He said: "fifty-fifty"." Why? "I don't know". "Are you sure you want this treatment to help you"? He said, "I do". "So how come", asked the therapist, that your involvement is incomplete? "I don't know" replied the client. Could it be, wondered the therapist, that your distress is too severe to prevent 
you of full participation? "I don't think so" was the answer. Do you feel, asked the therapist, that we can repeat the procedure that we took in the last sessions, re-experiencing again your traumatic event? The client said, "yes I can". "So, what do you think made it difficult for you to be fully involved in the task? Again, the client said "I don't know".

Having this dialogue stacked, the therapist made a switch and asked the client to describe his relationship with his son. The client replied: "he was my beloved, my friend and my pride, my heart" and burst into tears, stuttering "I caused his death... I didn't do enough to stop him from recruiting."

The therapist kept enquiring about this aspect of the event. "You said you did not agree to recruit him"? He said "yes". "Tell me, how did your son start thinking about the recruitment and what made him wishing to become a soldier?" He said, "I did everything to prevent him but he insisted and I was not able to refrain him from doing so". "So, you did all that and still couldn't prevent your son recruiting the army?" He said: "yes". "Do you feel that you failed?" "I had to try harder". "Do you feel guilty"? He said: "yes". "Do you feel that if you stopped suffering would mean you betray your sun?" He said: "nearly", and became silent. Then he burst into tears and murmured "yes, I agree. If I do not suffer, it means I am an unfaithful father". "So deep inside you feel that you should not gain relief from what we were doing in the treatment because you want to be faithful to your son?" He said: "yes". "So, we may conclude that you resist the treatment due to what you felt inside as immoral to relieve?" He said: "yes, I agree with you".

This dialogue represents the REBT therapy which emphasizes reducing symptoms of PTSD by changing negative to positive thoughts regards the traumatic event [22,24,29]. The appliance of TIR techniques met limits due to the patient's inner belief that giving up his suffer meant betraying his son. Only after revealing this negative (maladaptive) thought, the traumatic incident reduction could take place efficiently.

PSSI scores are presented in Table 1. The pre-intervention score of 43 indicated a severe symptom level of PTSD [37]. This score dropped drastically to 10 points on that scale after the first session and to 4 points after one month. Then it remained steadily on the 4 points level, which represents 'no symptom' level. These results are consistent with what is reported in the relevant literature when applying the TIR method [20,36,38].

\section{Discussion}

This study demonstrates the successful usage of combination of two approaches for the treatment of a traumatized person, namely, the TIR and the REBT. Application of these two approaches proved to be highly effective in alleviating symptoms of PTSD shortly after the commencement of the treatment, and to endure steadily in six months follow up, as measured by the PSSI scale

In the beginning of the therapeutic process TIR method was applied. However, throughout the sessions the therapist observed that despite seeming cooperation on the side of the patient, the symptoms were still present while the client trying to hide it; this is contrary to the scale results which apparently showed improvement of symptoms. This urged the therapist to look for the causes of the patient's inability to improve. An REBT dialogue was applied and found that the client was unwilling to improve because he related to his son with a moral linkage. The client felt that feeling better and recover from his PTSD symptoms would mean betrayal his son. The inner formulation was of the like "if I give up my suffer it shows I do not really care about my son; I would be a bad father". This inner belief was ameliorated by some guilt feelings concerning what he perceived his responsibility for his son`s death, because he did not do enough to prevent him from recruiting military service.

The therapist called this moral resistance. In other words, the client with the PTSD was unwilling to recover because of a moral obligation that expressed loyalty to his son.

The unstructured conversation had a significant effect in detecting the client's rejection of recovery and improvement from the PTSD symptoms. The conversation was more credible in decoding the inner feelings of the client since it relied not only on the client manifest wording, but also was attuned to other unintended signs such as body language and facial expressions.

Resistance in psychoanalysis refers to oppositional behavior when an individual's unconscious defenses of the ego are threatened by an external source. Sigmund Freud, the founder of psychoanalytic theory, developed his concept of resistance as he worked with patients who unexpectedly developed uncooperative behaviors during sessions of talk therapy. He reasoned that an individual that is suffering from a psychological affliction, which Freud believed to be derived from the presence of suppressed illicit or unwanted thoughts, may inadvertently attempt to impede any attempt to

Table 1: Results of Assessments.

\begin{tabular}{|l|c|c|c|c|c|c|}
\hline Severity of PTSD symptom & Pre-treatment & Week & One month & Three months & $\begin{array}{c}\text { Six months } \\
\text { Range of severity } \\
\text { PTSD }\end{array}$ \\
\hline Slight symptoms & & 10 & 7 & 4 & 10 \\
\hline Moderate symptoms & & & & 15 \\
\hline High moderate symptoms & & & & 20 \\
\hline High symptoms & & & & 25 \\
\hline Severe symptoms & & & & 30 \\
\hline Strongly severe symptoms & 43 & & & 35 \\
\hline
\end{tabular}

Note: This assessment according to PSSI scale represents the scores of PTSD pretreatment and post-intervention by TIR and REBT therapy. 
confront an unconsciously perceived threat. This would be for the purpose of inhibiting the revelation of repressed information from within the unconscious mind [39]. However, in this study, we found that the reason for resistance to therapeutic interventions was related to the client's unconscious need to keep preserving his mourning. We believe that moral resistance has to do with the cultural and social background in Arab countries, as many people believe that part of their loyalty to the deceased is to show sadness and to wear black. Due to this prevailing tradition in Arab countries they tend to reject psychological interventions which aid at relieving their grief, and prefer to be immersed in the sadness as an expression of their loyalty to the deceased, in addition, the culture of Arab countries plays a role in expressing the form of sadness, consistently with Walter who found that the expression of emotion by grieving Muslims in Egypt is very different from that of grieving Muslims in Bali. We believe that understanding the causes of treatment resistance is important in building a successful therapeutic relationship with the patient, and this agrees with Baker who claimed that understanding resistance as an aspect of the intersubjective field between therapist and patient, co-determined by both participants, greatly assists in the treatment of difficult patients. Resistance analysis in the case of a patient using religious references as resistance is presented here from an intersubjective perspective on psychodynamic treatment.

We consider the moral resistance to be a significant obstacle in treating PTSD symptoms, and it may be a paramount challenge for scientists and therapists when treating traumatized persons.

\section{Acknowledgments}

Special thanks to my colleagues Adam Said and Dr. Khaula Said and Dr. Osama Imad and Tsvi Gil and Umaia Said for their cooperation and encouragement.

\section{References}

1. Institute of Medicine. posttraumatic stress disorder, diagnosis, and treatment. National academies press, 500 fifth street, N.W. Lockbox 285, Washington. 2006.

2. Javidi H, Yadollahie M. Post-traumatic stress disorder. The International Journal of Occupational and Environmental Medicine. 2012; 3: 2-9.

3. American Psychiatric Association. Diagnostic and Statistical Manual of Mental Disorders, 5th ed. Arlington, VA: APA. 2013.

4. ACPMHMU, Logos N.Australian Guidelines for the Treatment of Adults with Acute Stress Disorder and Posttraumatic Stress Disorder. 2007.

5. Australian Center for Posttraumatic Mental health. Australian guidelines for the treatment of acute stress disorder \&posttraumatic stress disorder. 2013.

6. Ballenger JC, Davidson JR, Lecrubier Y, et al. Consensus statement update on posttraumatic stress disorder from the international consensus group on depression and anxiety. Journal of Clinical Psychiatry. 2004; 65: 55-62.

7. Bisson JI, Roberts NP, Andrew M, et al. Psychological therapies for chronic post-traumatic stress disorder (PTSD) in adults. Cochrane Database of Systematic Reviews. 2013; 12 .

8. Markowitz. Interpersonal Psychotherapy for Posttraumatic Stress Disorder. Oxford University Press. 198 Madison Avenue, New York, NY 10016, United States of America. 2017.

9. WHO. Investing mental health. Avenue Appia 20, 1211 Geneva 27, Switzerland. 2003.

10. Benight CC, Bandura A. Social cognitive theory of posttraumatic recovery: The role of perceived selfefficacy. Behavior research and therapy. 2004; 42: 1129-1148.

11. Shapiro F, Eye Movement Desensitization and Reprocessing (EMDR) Therapy. Basic Principles, Protocols, and Procedures. Third edition. The Guilford Press, A Division of Guilford Publications, Inc.370 Seventh Avenue, Suite 1200, New York, NY 10001.2018.

12. Walser R, Westrup D. Acceptance \& Commitment Therapy for the Treatment of Post-Traumatic Stress Disorder \& TraumaRelated Problems: A Practitioner's Guide to Using Mindfulness \& Acceptance Strategies. Oakland, CA: New Harbinger. 2007.

13. Raja S. Overcoming Trauma and PTSD, A Workbook Integrating Skills from ACT, DBT, and CBT. Library of Congress Cataloging-in-Publication Data. New Harbinger Publications, Inc. 5674 Shattuck Avenue, Oakland. 2012.

14. Cervone D, Pervien 1. Personality theory and research twelfth edition. John Wiley \& Sons, Inc. USA. 2013.

15. McLean CP, Foa EB. Prolonged exposure therapy for post-traumatic stress disorder: A review of evidence and dissemination. Expert review of neurotherapeutics. 2011; 11: 1151-1163.

16. Carbonell JL, Figley C. Running head: Promising PTSD Treatment Approaches A Systematic Clinical Demonstration of Promising PTSD Treatment approaches. Traumatology. 1999; 5: 32-48.

17. Valentine PV. Traumatic incident reduction: Brief treatment of trauma-related symptoms in incarcerated females.' Social Work Program School of Social and Behavioral Sciences the University of Alabama at Birmingham. 1998.

18. Descilo T, Fava NM, Burke S, et al. Effects of Traumatic Incident Reduction on Posttraumatic Symptoms in a Community-Based Agency. Research on Social Work Practice. 2019; 29: 401-409.

19. Valentine PV, Smith TE. Evaluating traumatic incident reduction therapy with female inmates: A randomized controlled clinical trial. Research on Social Work Practice. 2001; 11: 40-52.

20. Cukor J, Spitalnick J, Difede J, et al. Emerging treatments for PTSD. Clinical psychology review. 2009; 29: 715-726.

21. NREPP. Traumatic Incident Reduction. SAMHSA. National registry of evidence-based programs and practices. 2013; 734: 761-6268.

22. Boelen PA, de Keijser J, Smid G. Cognitive-Behavioral Variables Mediate the Impact of Violent Loss on Post-Loss 
Psychopathology. Psychological Trauma: Theory Research Practice and Policy. 2014; 7: 382-90.

23. Froggatt W. A brief introduction to rational emotive behavior therapy. Journal of Rational-Emotive and Cognitive Behaviour Therapy. 2005; 3: 115 .

24. Hyland P, Shevlin M, Adamson G, et al. Irrational beliefs in posttraumatic stress responses: A rational emotive behavior therapy approach. Journal of Loss and Trauma. 2015; 20: 171-188.

25. Ellis A, Dryden W. 2007 The practice of rational emotive behavior therapy. Springer publishing company.

26. Sorocco KH, Lauderdale S. Cognitive behavior therapy with older adults: Innovations across care settings. 2011.

27. Kwee M, Ellis A. The interface between rational emotive behavior therapy (REBT) and Zen. Journal of rational-emotive and cognitive-behavior therapy. 1998; 16: 5-43.

28. Terjesen MD, Kurasaki R. Rational emotive behavior therapy: Applications for working with parents and teachers. Estudos de Psicologia (Campinas). 2009; 26: 3-14.

29. Malkinson R. Cognitive-behavioral grief therapy: The ABC model of rational-emotion behavior therapy. Psihologijske teme. 2010; 19: 289-305.

30. David D, Cramer D. Rational and irrational beliefs in human feelings and psychophysiology. Rational and Irrational Beliefs. Research, Theory, and Clinical Practice. 2010; 96-112.

31. David D, Lynn SJ, Ellis A. Rational and irrational beliefs: Research, theory, and clinical practice. Oxford University Press. 2010.

32. Turner MJ, Slater MJ, Barker JB. Not the end of the world:
The effects of rational-emotive behavior therapy (REBT) on irrational beliefs in elite soccer academy athletes. Journal of Applied Sport Psychology. 2014; 26: 144-156.

33. Zepinic V. Treatment Resistant Symptoms of Complex PTSD Caused by Torture During War, Canadian Social Science, Canadian Academy of Oriental and Occidental Culture. 2015; 11: 9 .

34. Hembree EA, Foa EB, Feeny NC. 2002. Manual for the administration and scoring of the PTSD Symptom ScaleInterview (PSS-I). Unpublished manuscript available online at www.istss.org/resources/browse.com.;

35. Foa E, Riggs D, Dancu C, et al. Reliability and validity of a brief instrument for assessing post-traumatic stress disorder. Journal of Traumatic Stress. 1993; 6: 459-474.

36. Descilo T, Vedamurtachar A, Gerbarg P L, et al. Effects of a yoga breath intervention alone and in combination with an exposure therapy for post-traumatic stress disorder and depression in survivors of the 2004 South-East Asia tsunami. Acta Psychiatrica Scandinavica. 2017; 121: 289-300.

37. Freeman D, Thompson C, Vorontsova N, et al. Paranoia and post-traumatic stress disorder in the months after a physical assault: a longitudinal study examining shared and differential predictors. Psychological medicine. 2013; 43: 2673-2684.

38. Valentine P. V. Traumatic Incident Reduction I: Traumatized women inmates: Particulars of practice and research. Journal of Offender Rehabilitation. 2000; 31: 1-15.

39. Eid M, Larsen R.J. (Eds.). The science of subjective well-being. Guilford Press. 2008. 